\title{
Legionelosis ocupacional en mayores de 18 años: revisión sistemática
}

\author{
Occupational legionella in adults over 18 years pf age: \\ a systematic review
}

Andrea Domingo-Pueyo ${ }^{1}$ Javier Sanz-Valero ${ }^{1}$ Carmina Wanden-Berghe ${ }^{2}$
${ }^{1}$ Departamento de Salud Pública, Historia de la Ciencia y Ginecología de la Universidad Miguel Hernández de Elche. Campus de Sant Joan d'Alacant. Alicante Elche España.jsanz@umh.es ${ }^{2}$ Hospital General de Alicante. Alicante Elche España.

\begin{abstract}
The scope of this paper is to review the available scientific literature about the effects on health of occupational exposure to Legionella spp. A systematic review of the scientific literature retrieved from the MEDLINE (via PubMed), ISIWeb of Science (Institute for Scientific Information), Cochrane Library, LILCAS and SCOPUS databases through February 2015 was conducted. The key words used were "«Legionnaires' Disease» «Legionellosis» «Occupational Exposure» «Occupational Diseases». Two hundred and twenty-two references were retrieved of which, after applying inclusion/exclusion criteria, 13 complete articles were selected. Of these, 9 describe pneumonia, 4 list Pontiac Fever, 1 reveals legionellosis and 3 result in death. The main causative agent of disease in workers exposed was Legionella pneumophila serogroup 1. Legionella spp infection is closely related to professions where there is nebulization with water, mainly among workers subjected to long exposure or even re-exposure. Outbreaks occurs seasonally, especially in the hottest months.
\end{abstract}

Key words Legionnaires' disease, Legionellosis, Occupational exposure, Occupational diseases
Resumen El objetivo es revisar la literatura cientifica sobre los problemas de salud derivados de la exposición laboral a Legionella spp. Revisión sistemática de la literatura científica recogida en las bases de datos MEDLINE (Pubmed), ISI-Web of Science (Institute for Scientific Information), Cochrane Library Plus, Literatura Latinoamericana de Información en Ciencias de la Salud (LILACS) y SCOPUS, hasta febrero 2015. Los descriptores utilizados fueron: "Legionnaires' Disease" "Legionellosis" "Occupational Exposure" "Occupational Diseases". Se obtuvieron 222 referencias, que tras aplicar los criterios de inclusión $y$ exclusión, se seleccionaron 13 artículos a texto completo. De ellos 9 artículos describen la aparición de neumonía, 4 Fiebre de Pontiac, 1 legionelosis o presunta legionelosis y 3 muerte. El principal agente causal en los profesionales expuestos fue Legionella pneumophila serogrupo 1. La infección por legionela está ligada a las profesiones donde existe nebulización por agua, principalmente en los trabajadores sometidos a largas exposiciones o incluso a la re-exposición. Los brotes se producen de forma estacional, sobre todo en los meses más cálidos.

Palabras clave Enfermedad de los legionarios, Legionelosis, Exposición profesional, Enfermedades profesionales 


\section{Introducción}

La legionelosis es un término genérico que describe la Enfermedad del Legionario y la Fiebre de Pontiac $^{1}$. La primera se trata de una enfermedad sistémica con neumonía como mayor fallo prominente, y la segunda, es una enfermedad autolimitada con una tasa de ataque alta, caracterizada por fiebre, dolor de cabeza, mialgia y fatiga ${ }^{2}$, en la que los casos presentan síntomas similares en un periodo corto de tiempo seguido de una exposición a una misma fuente ${ }^{3}$. El primer brote de Fiebre de Pontiac afectó a 144 personas en un centro de salud en Pontiac, Michigan en 1968 (retrospectivamente descrito en 1978) ${ }^{1}$.

El principal agente causante de la legionelosis, Legionella pneumophila, ha sido reconocido como un importante generador de neumonía, responsable de la mayoría de los brotes en EEUU y Europa ${ }^{4}$. Se estima que en EEUU, Legionella spp, provoca entre 8000-18000 casos de neumonía por legionela en un año ${ }^{5}$. En 2007 y 2008, un total de 11897 casos de legionelosis fueron diagnosticados en Europa ${ }^{3}$. Los brotes han sido detectados en muchas comunidades, siendo el mayor de ellos el registrado en Europa en 1996, con más de 200 personas afectadas por Legionella pneumophilla serogrupo $1^{6}$. Son infecciones relativamente poco comunes, con alrededor de 200 casos registrados anualmente en Inglaterra y Gales, aunque la tasa de registro es del $10 \%$ puesto que los síntomas pueden ser confundidos con otro tipo de neumonía $^{1}$. España, sin embargo, es el país con el mayor número de casos declarados ${ }^{6}$. Durante el quinquenio 2002-2006 el Instituto Municipal de Salud Pública de Barcelona registró un promedio de 21 casos anuales de neumonía por Legionella notificados (Rango 13-34) ${ }^{7}$.

Estas enfermedades son causadas por la inhalación de gotículas de agua o partículas que contienen la bacteria termófila de la familia $L e$ gionellaceae, que son alrededor de 40 especies. La más comúnmente asociada con la Enfermedad el Legionario es la Legionella pneumophila serogrupo 1 , de las 14 existentes $^{1}$. La Legionella pneumophila, agente etiológico de la legionelosis, aparece por estar ampliamente en un entorno natural húmedo y ha sido encontrado en aguas naturales no asociadas a enfermedad, a menudo en concentraciones de 106 organismos/litro, a temperaturas desde $5-65^{\circ} \mathrm{C}^{8}$. El calor y la humedad del periodo estival con una media de temperatura superior a $20^{\circ} \mathrm{C}$ favorecen la proliferación de las bacterias 9 . Esta pequeña bacteria penetra profundamente en el alveolo, lugar donde causa la infección. Tiene un periodo de incubación entre 2-10 días previos a la aparición de los síntomas. Para la Fiebre de Pontiac pueden desarrollarse desde 5 horas a 3 días tras la exposición ${ }^{10}$.

Aunque el modo de transmisión no se conoce siempre, la evidencia del rol de la inhalación de agua aerosolizada contaminada, es el principal causante ${ }^{8,11}$. A pesar de que en la población en general la exposición está principalmente ligada a la estancia en hoteles, hospitales ${ }^{12}$ y spas, en los trabajadores, se ha asociado a sistemas de agua caliente, sistemas de torre de refrigeración usados en aires acondicionados, refrigeradores industriales utilizados en la lubricación de la maquinaria, la limpieza de las turbinas de los condensadores con aire comprimido y suministro de agua municipal ${ }^{9}$, aunque también se consideran factores de riesgo la exposición a excavaciones y construcciones ${ }^{4}$.

Dicho lo cual, sabiendo que la exposición a Legionella spp es perjudicial para la salud, el objetivo del presente estudio es revisar la literatura científica sobre los efectos en la salud de los trabajadores expuestos a este agente biológico.

\section{Métodos}

Se realizó análisis crítico y sistemático de los trabajos recuperados mediante revisión de la literatura científica.

Los datos utilizados en este estudio se obtuvieron de la consulta directa y acceso vía Internet de la literatura científica recogida en las siguientes bases de datos: MEDLINE (vía PubMed), ISIWeb of Science (Institute for Scientific Information), Cochrane Library Plus, Literatura Latinoamericana de Información en Ciencias de la Salud (Lilacs) y SCOPUS.

Para la recuperación documental se empleó el Thesaurus desarrollado por la U.S. National Library of Medicine. No se utilizaron Calificadores de Materia (Subheadings), ni fue necesario el empleo de Tags. De su estudio se consideraron adecuados los términos "Legionnaires' Disease", "Legionellosis", "Occupational Exposure", "Occupational Diseases" tanto como descriptores como en formato texto en el título y resumen. La ecuación de búsqueda final se desarrolló para su empleo en la base de datos MEDLINE, vía PubMed, mediante la utilización de los conectores booleanos:

("Legionnaires' Disease" [Mesh] OR "Legionnaires' Disease" [Title/Abstract] OR "Legionellosis"[Mesh] OR "Legionellosis"[Title/Abstract]) 
AND ("Occupational Exposure"[Mesh] OR "Occupational Diseases”[Title/Abstract])

Se utilizaron los filtros (límites): "Humans", "Adult (more than 18 years)". Adaptándose posteriormente a las bases de datos anteriormente mencionadas.

La búsqueda se realizó desde la primera fecha disponible, de acuerdo a las características de cada base de datos, hasta febrero 2015 (momento de la última actualización). Adicionalmente, como búsqueda secundaria y para reducir el número de trabajos no recuperados, se examinó el listado bibliográfico de los artículos que fueron seleccionados en la búsqueda principal, con el objeto de identificar estudios no detectados en la revisión.

La elección final de los artículos se realizó según el cumplimiento de los siguientes criterios:

Inclusión: Los documentos debían ser estudios observacionales publicados en revistas revisadas por pares, seleccionándose aquellos pertinentes cuyo texto completo pudo ser recuperado, debiendo estar escritos en inglés, portugués o castellano.

Exclusión: Se excluyeron los artículos que no centraban la intervención en los efectos para la salud humana de la exposición laboral a $L e$ gionella spp (existencia de relación causa-efecto entre exposición y síntoma/patología) y los que incluían individuos menores a 18 años en sus estudios.

La selección de los artículos pertinentes se realizó de forma independiente por dos de los autores de la presente revisión (D-P y S-V). Para dar por válida la elección de los artículos para la revisión se estableció que la valoración de la concordancia entre estos dos autores (índice Kappa) debía ser superior a 0.80 (medida de la fuerza de la concordancia muy buena ${ }^{13}$. Siempre que se cumpliera esta condición, las posibles discordancias se solucionarían mediante la consulta a la tercera autora $(\mathrm{W}-\mathrm{B})$ y posterior consenso entre los autores.

La calidad de los artículos seleccionados se realizó de forma conjunta tomando como soporte las directrices para la comunicación de estudios observacionales STROBE (STrengthening the Reporting of OBservational studies in Epidemiology $)^{14}$, que contiene una lista de 22 puntos esenciales que deben describirse en la publicación de estos estudios. Para ello, se puntuó cada ítem en función de si en el artículo estaba recogida " 1 " o no " 0 " la información. En el caso de no ser necesaria la evaluación de algún ítem ese punto no era contabilizado en el total (No Aplica
$=$ NA). Cuando un ítem estaba compuesto por varios puntos, estos se evaluaron de forma independiente, dándole el mismo valor a cada uno de ellos y posteriormente se realizó un promedio (siendo éste el resultado final de ese ítem), de tal forma que en ningún caso se pudiera superar la puntuación de 1 punto por ítem.

El control de la información extraída de los estudios revisados se realizó mediante tablas de doble entrada que permitían la detección de los errores y la corrección mediante nueva consulta de los originales. Los artículos se agruparon según las variables a estudio, con el fin de sistematizar y facilitar la comprensión de los resultados, codificando los siguientes datos: primer autor de la referencia bibliográfica y año de publicación, diseño del estudio, población del estudio, profesión, fuente de exposición, tiempo de exposición previa a la sintomatología, aparición del brote, bacteria, tratamiento, país donde se realizó el estudio y efecto de la exposición.

\section{Resultados}

Con los criterios de búsqueda descritos se recuperaron 222 referencias de las que tras depurar los duplicados y aplicar los criterios de inclusión y exclusión (Figura 1), fue posible recuperar a texto completo 13 artículos $(1-9,11,12,15,16)$ (Tabla 1), procedentes de Medline ( $\mathrm{n}=9,69,23 \%)$, SCOPUS ( $\mathrm{n}=4,30,77 \%)$, Web of Science, Cochrane y Lilacs $(\mathrm{n}=0,0 \%)$. El acuerdo sobre la pertinencia de los estudios seleccionados entre los dos evaluadores fue del 100\%. Al analizar la calidad de los artículos seleccionados para la revisión, mediante el cuestionario $\mathrm{STROBE}^{14}$, las puntuaciones oscilaban entre 8,07 y 17,47 (mediana $=14,13)($ Tabla 2).

El diseño de todos los trabajos revisados fueron informes de $\operatorname{casos}^{1-4,6-9,11,15,16}$, excepto 2 que fueron casos y controles ${ }^{5,12}$. El trabajo de mayor tamaño muestral lo describió Fry et al. ${ }^{5}$ con 17 individuos y el de menor Allen et al. ${ }^{2}$ con sólo 1. La población estudiada en 6 de los 13 trabajos estaba formada exclusivamente por hombres $^{3,8,11,12,15,16}$ y en 1 por hombres y mujeres ${ }^{1}$. En el resto de trabajos no se especifica el sexo ${ }^{2,4-7,9}$. Sólo en 1 estudio se observó población mayor a 65 años 5 .

La fecha de publicación de los trabajos revisados oscilaba entre 1982 y 2010. Los países en los que se llevaron a cabo un mayor número de estudios fue EEUU ${ }^{5,15,16}$ con 3 artículos, seguido de España ${ }^{6,7}$ e Italia ${ }^{4,8}$ con dos trabajos cada uno. 


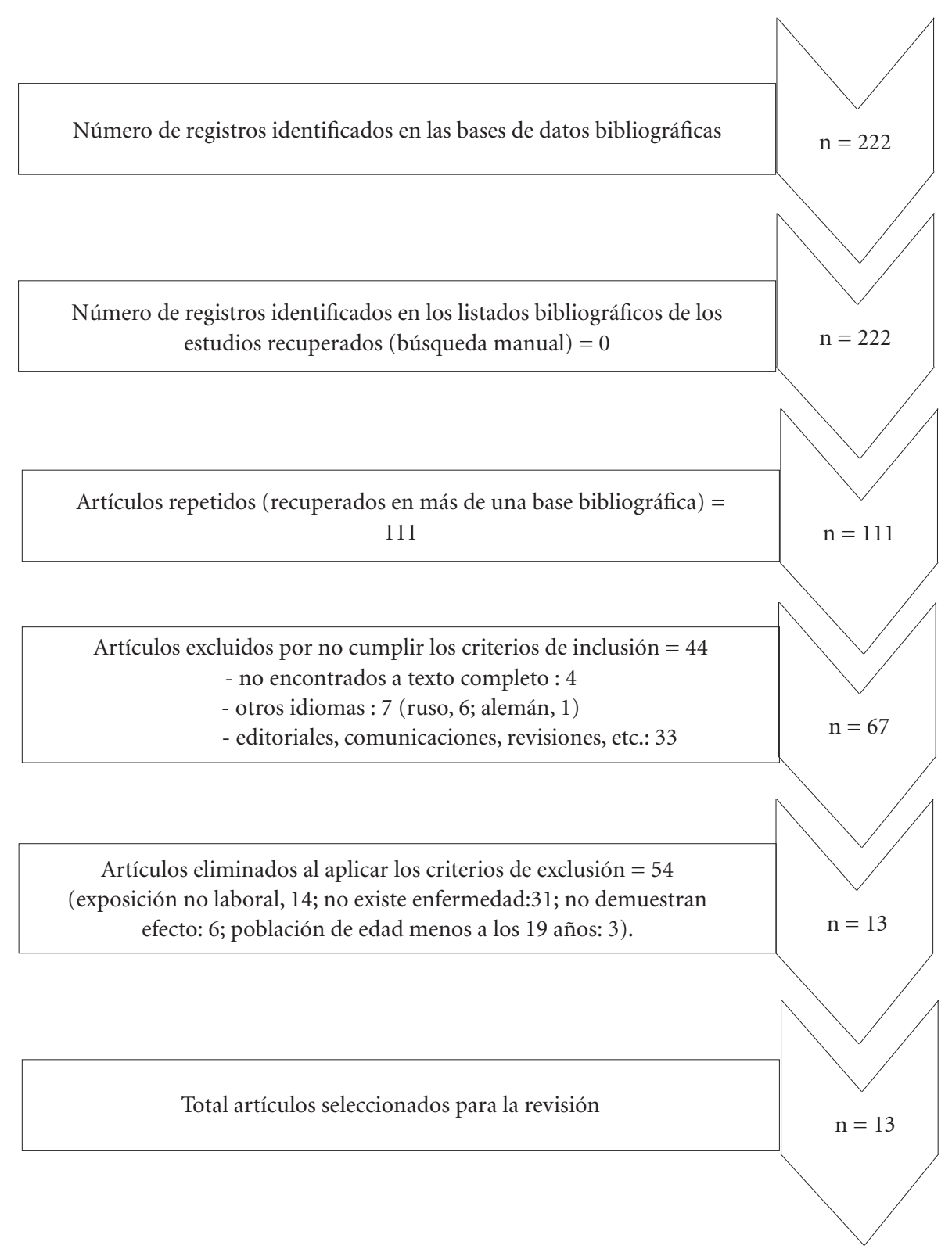

Figura 1. Diagrama de selección de artículos incluidos en la revisión: legionelosis ocupacional en mayores de 18 años.

El resto de países revisados sólo fueron motivo de publicación una vez: Finlandia ${ }^{3}$, Nueva Zelan$\mathrm{da}^{1}$, Japón $^{11}$, Dinamarca ${ }^{9}$ y Reino Unido ${ }^{12}$. Allen et al. $^{2}$ no especifican el país donde tuvo lugar su estudio.

La mayoría de los casos tuvieron lugar durante los meses más cálidos (marzo-septiembre $)^{3,7,9,11,15,16}$, aunque también se registraron en meses fríos: enero ${ }^{1,6,8}$, febrero ${ }^{2}$, marzo ${ }^{5}$ y octu- bre $^{4}$. En el caso de Morton et al. ${ }^{12}$, el brote tuve lugar entre los meses de septiembre y octubre. El agente causal más frecuentemente reconocido fue Legionella pneumophila serogrupo $1^{2,5-8,12,15,16}$. Cramp et al. ${ }^{1}$ identificaron Legionella longbeachse serogrupo 2 como fuente de infección. En el caso de Kusnetsov et al. ${ }^{3}$ fueron varias las bacterias aisladas: Legionella pneumophila serogrupo 1-4, Legionella micdadei y legionella dumoffi. Sin 


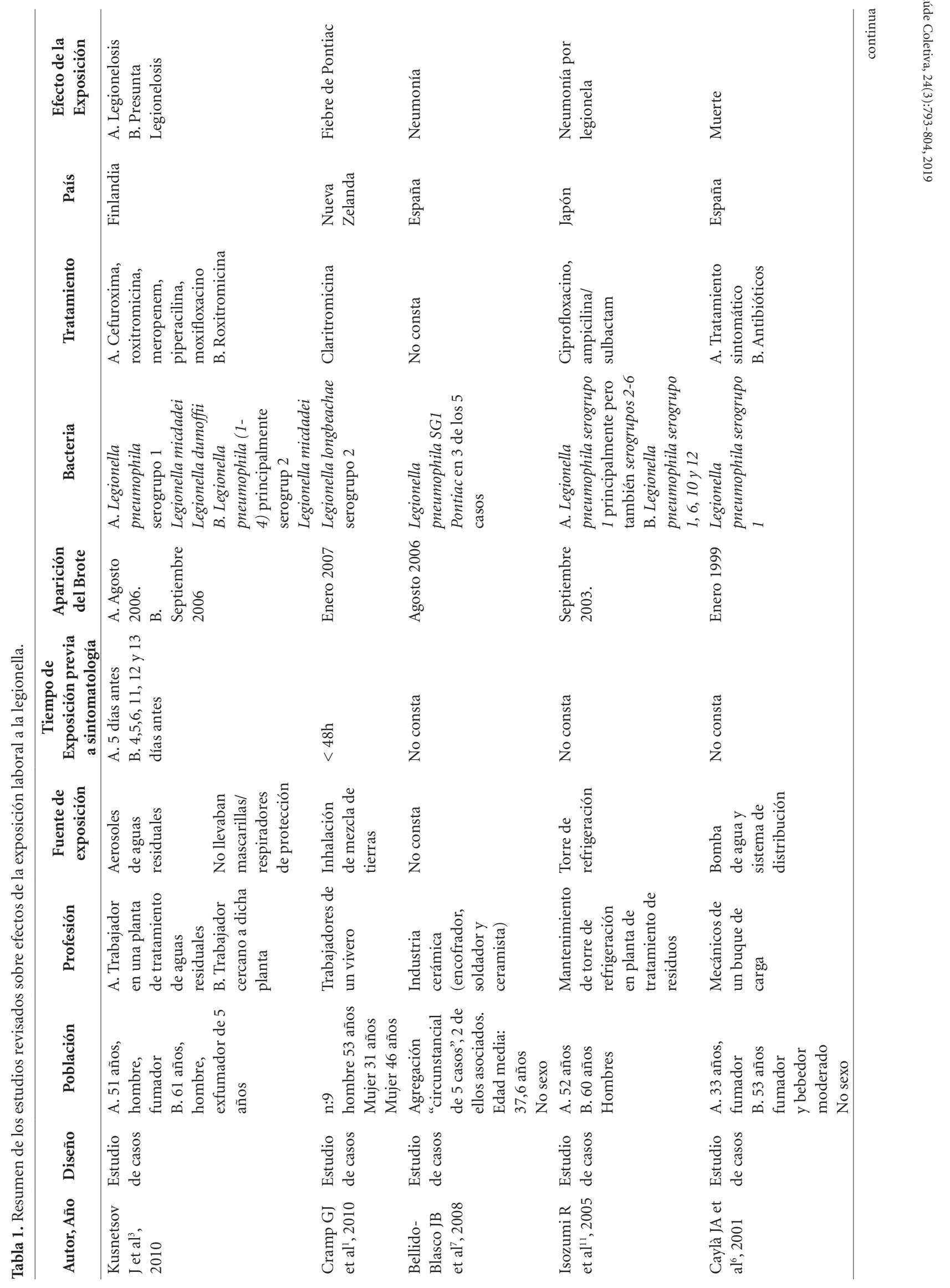




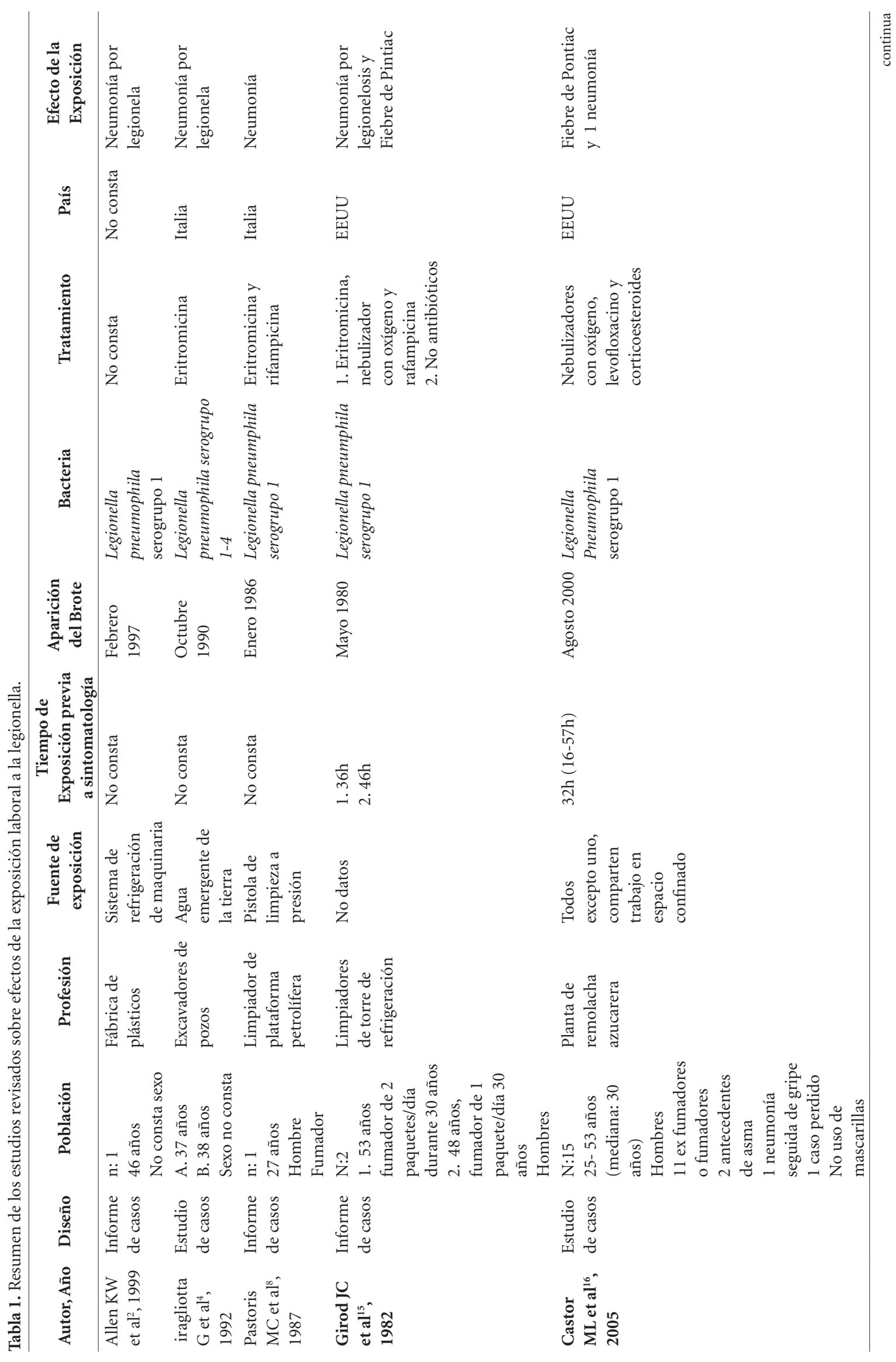




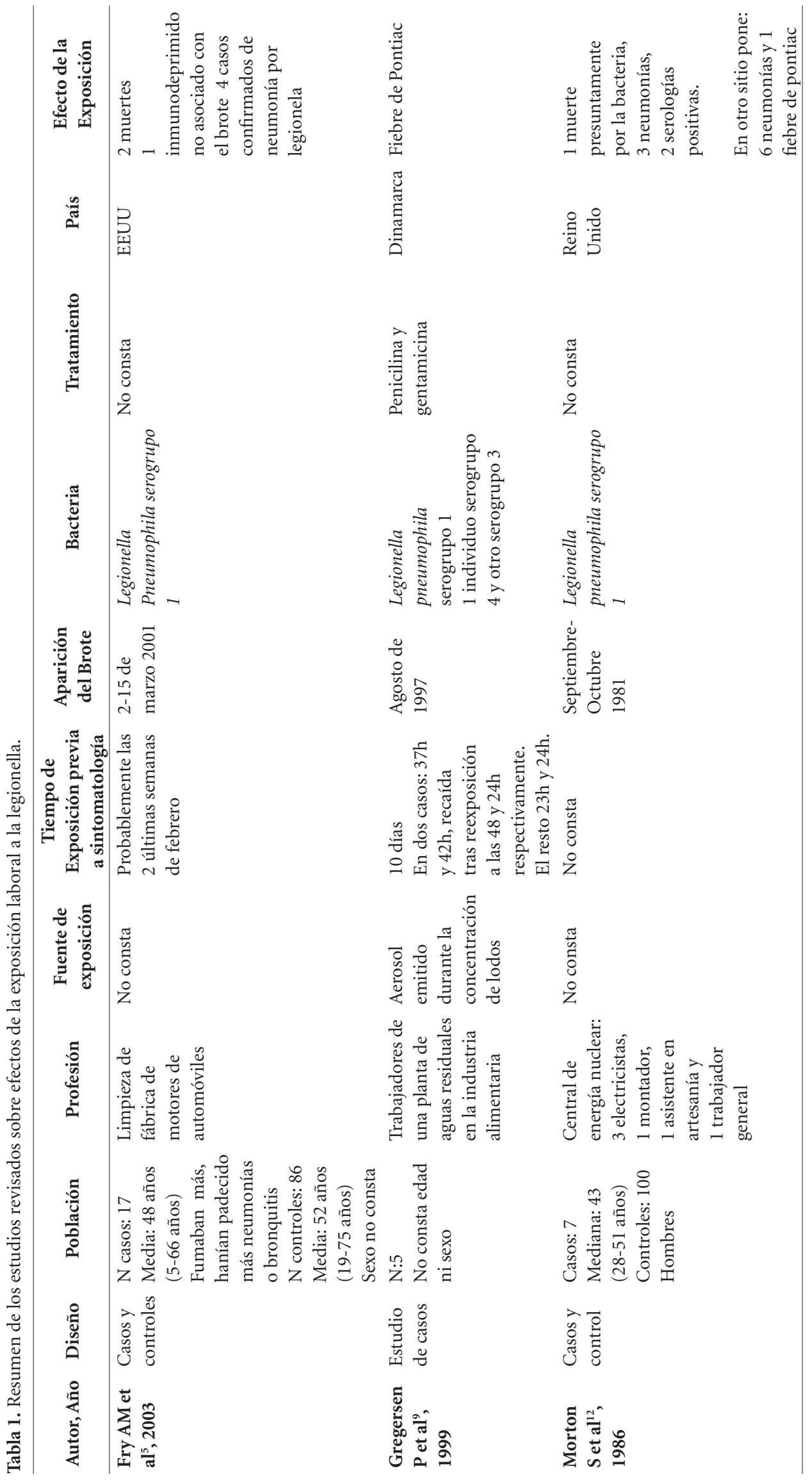


embargo, en los trabajos de Miragliotta et al. ${ }^{4} \mathrm{y}$ Isozumi et al. ${ }^{11}$ hallaron Legionella pneumophila serogrupo1-4, y éste último además serogrupo 5 y 6 . En el estudio de Gregersen et al. ${ }^{9}$ sólo se mostró Legionella pneumophila serogrupo 3 y 4. Estas bacterias provocaron en su mayoría neumonías ${ }^{2,4,5,7,8,11,12,15,16}$, aunque también originaron: Fiebre de Pontiac ${ }^{1,9,15,16}$, legionelosis o presunta legionelosis ${ }^{3}$ incluso la muerte ${ }^{5,6,12}$. El tiempo de exposición previo a la aparición de síntomas osciló entre $32 \mathrm{~h}^{16}$ y $5^{3}$ días. En todos los casos en los que se ha descrito la fuente de exposición intervino agua aerosolizada ${ }^{2-4,6,8,9,11}$ excepto en el caso de Cramp et al. ${ }^{1}$ en el que la inhalación fue a través de una mezcla de tierras. En el resto de los artículos revisados no consta la fuente de exposición ${ }^{5,7,12,15,16}$. Las profesiones a las que se dedicaban los individuos a estudio fueron muy desiguales: empleados en una planta de tratamiento de aguas y residuos ${ }^{3,9,11}$, trabajadores de un vivero ${ }^{1}$, de la industria cerámica ${ }^{7}$, de una fábrica de plásticos ${ }^{2}$, de una fábrica de remolacha azucarera ${ }^{16}$, fábrica de motores de coches $^{5}$, de una plataforma petrolífera ${ }^{8}$, de una central de energía nuclear ${ }^{12}$, excavadores de pozos $^{4} \mathrm{y}$ empleados de un buque de carga ${ }^{6}$. A pesar de que los empleos fueron tan dispares, la profesión que ejercían dentro de dichas industrias en 3 casos coincidió en trabajos de limpieza ${ }^{5,8,15}$.

El tratamiento para las infecciones fue heterogéneo. En algunos casos se prescribieron antibióticos: macrólidos ${ }^{1,3,4,8,15}$, betalactámicos ${ }^{3,9,11}$, quinolonas $^{3,11,16}$, aminoglucósidos ${ }^{9}$ y rifamicinas ${ }^{8,15}$; en otros: se centraron en el tratamiento sintómatico ${ }^{6,15}$ y Morton et al. ${ }^{12}$ no administraron ningún tratamiento. En el caso de Castor et al. ${ }^{16}$ el tratamiento antibiótico fue acompañado de corticoesteroides y nebulizadores, y en el de Girod et al. ${ }^{15}$ como tratamiento complementario también se utilizaron nebulizadores. Los trabajos de Bellido-Blasco et al. ${ }^{7}$, Allen et al. ${ }^{2}$ y Fry et al. ${ }^{5}$ no explicitaron el tipo de tratamiento llevado a cabo para la sanación de los pacientes. $\operatorname{Otros}^{3,8,9,11,15}$, administran varios tratamientos antimicrobianos complementarios.

\section{Discusión}

Podría parecer que la legionelosis está más comúnmente asociada al área recreacional (hoteles, spas, etc.) que al laboral, sin embargo, la presente revisión demuestra que la infección por Legionella spp está íntimamente ligada a ciertas profesiones, sobre todo en aquellas en las que se utilizan 
torres de refrigeración, condensadores evaporativos o limpiezas con agua a presión. Es decir, trabajos relacionados con la nebulización.

Hay que prestar especial atención al hecho de que de la base de datos Web of Science, no haya sido incluida ninguna referencia; el motivo es que de las 91 referencias encontradas, 73 estaban repetidas en alguna de las otras dos fuentes interrogadas, 8 no trataban sobre exposición laboral, 9 no eran artículos originales y 1 no trataba sobre la enfermedad.

Coincidiendo con nuestra revisión, según el Centro Nacional de Epidemiología del Instituto de Salud Carlos III $^{17}$, para todos los grupos de edad, la incidencia de legionelosis ha sido mayor en hombres que en mujeres. Ajustándose también a nuestros resultados, esta misma fuente en otro trabajo publicado ${ }^{18}$, muestra que los brotes tienen una presentación estacional, con un mayor número en los meses comprendidos entre abril y septiembre, seguidos de los meses de octubre y enero respectivamente. Bellido-Blasco et al. ${ }^{7}$ ofrecen como posible hipótesis para la aparición de Legionella spp en el medio laboral, que la influencia del ritmo de la actividad industrial en agosto, parada y nueva puesta en marcha, pudo tener relación con la cronología de su episodio, significando un riesgo general en ese tipo de polígonos industriales en los que la presencia de torres de refrigeración es relativamente alta. Se puede pensar que un mayor número de casos recogidos en esta revisión en países de zonas cálidas, hecho que probablemente se deba a sus temperaturas cosa que facilita la proliferación de la bacteria ${ }^{1,4,6-8}$.

No podemos mostrar estas mismas coincidencias al referirnos a los rangos etarios, pues parece existir una relación lineal directa entre edad- incidencia ${ }^{17}$. Nuestras diferencias podrían deberse a que poblaciones de individuos mayores de 65 años (sólo se ha recogido un artículo en esta revisión), están apartados de la vida laboral. Para el resto de rangos no es posible realizar una comparativa pues no se dispone de la edad de todos los individuos incluidos en cada artículo.

En la mayoría de los casos, la bacteria causal de la enfermedad fue Legionella pneumophila serogrupo 1. Ocurre lo mismo para los 28 brotes de los 501 registrados en España entre 1999-2009, en los que se pudieron genotipar las cepas clínicas y ambientales que concluyeron que fueron idénticas en el estudio de comparación genético realizado en el laboratorio de referencia ${ }^{19}$. Contrariamente a lo que ocurre en España, algunos casos de la Enfermedad de los Legionarios, han sido causados por L. longbeachae representando el 50\% de los casos en Nueva Zelanda y el 30\% de los adquiridos en Australia ${ }^{1}$. Cabe tener en cuenta, que ciertas amebas pueden actuar como huésped/reservorio de la gram negativa pudiendo ser el agente etiológico de la Fiebre de Pontiac tal y como indica uno de nuestros resultados ${ }^{16}$ coincidiendo con la revisión de Rowbotham ${ }^{20}$. El caso de Castor et al. ${ }^{16}$ observaron una alta cantidad de endotoxinas, que mostró la alta concentración de bacterias gram negativas que se hallaba en el agua de limpieza en la planta de remolacha azucarera, pudiendo ejercer un rol fundamental en la infestación.

Es curioso el caso que describen Pastoris et al. ${ }^{8}$ pues tiene lugar en una plataforma petrolífera que se supone un entorno completamente aislado, en el que los sistemas de agua suplidos por agua desalinizada no ha mostrado resto de infección por Legionella spp. En otros estudios, sospechan de la capacidad del viento para transportar la bacteria y ser fuente de infección ${ }^{3}$. En el caso de Bellido-Blasco et al. ${ }^{7}$, de los 5 casos coexistentes, sólo se pudieron agregar dos como producidos por un mismo agente, pues parece ser que los otros tres simplemente coincidieron en el tiempo, o el caso de Fry et al. ${ }^{5}$ en el que no se encontró ninguna muestra medioambiental que concordase, ni ninguna muestra de los participantes del estudio que probasen la exposición aguda a Legionella spp. Este es un problema que se ha podido observar por varios autores, en los que no se han podido identificar en todas las muestras fisiológicas de los empleados ni en las ambientales, además de la dificultad para aislar la bacteria en los entornos laborales ${ }^{7}$; y esto es de especial importancia puesto que es necesaria la determinación de títulos de anticuerpos para evaluar los riesgos de la infección ya que estos se encuentran elevados aún en individuos sanos ${ }^{11} \mathrm{o}$ individuos que han estado expuestos en algún momento a la bacteria ${ }^{21}$. Además, cultivos y muestras de orina de los individuos enfermos son comúnmente negativos en los brotes de Fiebre de Pontiac. Es posible que la administración temprana de esteroides y antibióticos intravenosos enmascare la respuesta inmune de los individuos ${ }^{16}$.

La virulencia de la cepa así como la concentración de la misma, el tamaño de partícula inhalada ${ }^{15}$, la susceptibilidad y mecanismos de defensa del huésped, la larga duración de las jornadas de trabajo, largos periodos de exposición a aguas aerosolizadas contaminadas o reexposiciones ${ }^{9}$, lugares de trabajo confinados ${ }^{16}$ y la falta de protección respiratoria ${ }^{16}$, puede jugar parte en el 
proceso de infección, porque tal y como exponen Pastoris et al, 9 trabajadores estuvieron expuestos a las mismas condiciones que los infectados y no mostraron ningún signo clínico ni serológico de infección. En su caso, el organismo podría no haberse multiplicado activamente a temperaturas en las que el agua estaba almacenada pero podría ciertamente haber sobrevivido. No se conoce si la exposición a concentraciones relativamente bajas de L pneumophila durante largos periodos de tiempo superiores a $12 \mathrm{~h}$ podría originar la infección a personas susceptibles ${ }^{8}$. Se ha teorizado sobre la fiebre de Pontiac como resultado de una exposición de organismos de Legionella spp no viables. Esto podría explicar el fallo a la hora de detectarlo como agente infeccioso viable o al observar el proceso de la enfermedad ${ }^{16}$. Más significativa aún es la hipótesis evidenciada de Girod et al. ${ }^{15}$ en el que los 2 trabajadores estuvieron expuestos a un inóculo que presumiblemente estaba formado por la misma cantidad de organismos vivos y muertos y como resultado uno de ellos presentó la forma grave de legionelosis y el otro la leve.

El hecho de que los tratamientos hayan sido tan dispares, da una idea de la dificultad del diagnóstico temprano, pues en ciertos casos parece que se ha confundido con tuberculosis ${ }^{8,15}$, o con un simple resfriado en el que la prescripción ha sido meramente sintomática ${ }^{6}$. Esta dificultad de diagnóstico, sobretodo en el caso de Fiebre de Pontiac, hace más complicada la notificación y posible identificación de los casos.

Posibles limitaciones de esta revisión: Los diseños observados en los estudios revisados -casos y controles e informe de casos-, aportan un nivel de evidencia máximo IIb y grado de recomendación B, según la US Agency for Health Research and Quality, y en consecuencia que las conclusiones alcanzadas y más aún, la aplicabilidad de las intervenciones apoyadas en los estudios observacionales, sean poco sólidas. Pero, hay que tener presente el tema a estudio -exposición a Legionella spp-y por tanto asumir que no es posible aspirar a diseños de mayor nivel y grado de recomendación ${ }^{22}$. Aunque las revisiones sistemáticas se deben basar en estudios con seguimientos y diseños que garanticen el mayor rigor científico, en el presente análisis se incluyeron todos los artículos que trataron el tema analizado para alcanzar la máxima representación en los resultados.

La mayoría de los casos de legionelosis ocurren esporádicamente y no están asociados a una fuente de infección o un brote ${ }^{21}$. Así los casos esporádicos tienen una baja notificación a las au- toridades sanitarias (en EEUU se estima que sólo se notifica un 5\% de los casos), sin embargo, los brotes, normalmente tienen un impacto medio mayor ${ }^{6}$. En España la Red Nacional de Vigilancia Epidemiológica obliga a su notificación. Sin embargo, a pesar de dicha obligatoriedad, existe una infrapublicación en revistas científicas debido, quizá, a la mala prensa que origina un brote en una cierta comunidad, causa que ha imposibilitado recopilar un número mayor de artículos que versen sobre el tema a estudio.

Considerando la mayoría de los diseños recopilados en este trabajo, es probable que el cuestionario STROBE no sea el más indicado para evaluar la calidad de los artículos, es por ello que el valor obtenido en los resultados no es tan alto como se espera de estudios rigurosos. Sin embargo, se ha tratado de paliar dicha limitación, pues no hay constancia de que exista ningún otro cuestionario que evalúe la calidad de los artículos observacionales, acoplando los valores absolutos obtenidos a valores relativos, no computando aquellos puntos en los que no aplicaba su exposición ${ }^{22}$.

Ciertos artículos interesantes no han podido ser incluidos en esta revisión por no cumplir los criterios; no obstante cabe señalar que la exposición a Legionella spp también se dio, entre otros, en soldadores ${ }^{23}$ a través del sistema de refrigeración de la soldadura, en industrias con tanques utilizados para el pretratamiento de pinturas ${ }^{24}$, en trabajadores de mantenimiento de un hospi$\mathrm{tal}^{25}$, o en dentistas.

Además de las limitaciones que sufre esta revisión, se debe hacer referencia a las que tienen ciertos artículos recogidos como resultado de este estudio: Miragliotta et al. $^{4}$ no realizaron un control complementario sobre el agua de uso doméstico, pues lo descartaron como origen de infección ya que los empleados infectados no vivían en el mismo vecindario y puesto que no pudieron describir el modo exacto de transmisión, ciertamente debieron descartarlo como posible foco; en el caso de Fry et al..$^{5}$ los casos fumaban más que lo controles y habían sufrido más neumonía o bronquitis; en ciertos trabajos no se hayan incluido sexo y/o edad $\mathrm{d}^{2,6,7}$.

Para preservar el estado completo de salud de los trabajadores es necesario reconocer que las aguas de las plantas de procesamiento industrial pueden estar contaminadas con Legionella spp y que el lugar de trabajo puede entrañar peligro para los trabajadores ${ }^{11}$. Así pues, la legionelosis debe ser considerada en el diagnóstico diferencial de personas que trabajen en entornos industria- 
les y que presenten enfermedad respiratoria febril aguda con síntomas sistémicos, especialmente en aquellos que presenten enfermedades crónicas y sean fumadores ${ }^{5,25}$. Puesto que el organismo se encuentra ampliamente en agua y suelos, ningún sistema de agua que pueda generar aerosoles debe ser descartado como posible fuente/reservorio $^{2}$. En los lugares donde existe riesgo de legionelosis deben mantenerse los equipos y sistemas lo más limpios posibles y ser desinfectados regularmente. Si es posible, el agua debe mantenerse por debajo de los $20^{\circ} \mathrm{C}$ o por encima de los $50^{\circ} \mathrm{C}$. Si no es posible, deben añadir biocidas al agua para prevenir la multiplicación bacteria$n a^{10}$. Está demostrado que los biocidas muestran una efectividad in vitro pero que no erradican el organismo si la rutina de mantenimiento no se lleva a cabo ${ }^{12}$. Asimismo, la exposición a Legionella spp puede ser eliminada o significativamente reducida utilizando mascarillas como parte del equipo de protección personal, utilizando una buena ventilación en el lugar y en el caso de viveros, almacenando la mezcla de tierras en áreas refrigeradas ${ }^{1}$.
Por todo lo anteriormente expuesto, se puede concluir que las medidas para la conservación de la salud deben empezar al principio de la formación en un puesto de trabajo. La infección se puede disminuir significativamente manteniendo un ambiente limpio y utilizando equipos de protección personal, como las mascarillas. Además, y si se tiene en cuenta que un individuo permanece de media un tercio de su vida en su lugar de trabajo se puede interpretar que la exposición a Legionella spp produce alteraciones en humanos con resultados de diferente relevancia y por ello se deben emplear medidas de seguridad adecuadas en el entorno laboral con el fin de minimizar dicha exposición y por consiguiente disminuir el número de patologías asociadas a la misma.

La infección por Legionella spp está íntimamente ligada a las profesiones donde existe nebulización (aerosoles) por agua, principalmente en los trabajadores sometidos a largas exposiciones o incluso a la re-exposición. Los brotes se producen de forma estacional, sobre todo en los meses más cálidos.

\section{Colaboradores}

Los tres autores han participado simultáneamente en la concepción y el diseño del trabajo, la recogida de datos, el análisis y la interpretación de los datos. Así como en la escritura del artículo, la revisión crítica con contribución intelectuales y en la aprobación de la versión final para su publicación y que asumen la responsabilidad de la integridad del trabajo en su conjunto. 


\section{Referencias}

1. Cramp GJ, Harte D, Douglas NM, Graham F, Schousboe M, Sykes K. An outbreak of Pontiac fever due to Legionella longbeachae serogroup 2 found in potting mix in a horticultural nursery in New Zealand. Epidemiol Infect 2010; 138(1):15-20.

2. Allen KW, Prempeh H, Osman MS. Legionella pneumonia from a novel industrial aerosol. Commun Dis Public Health PHLS 1999; 2(4):294-296.

3. Kusnetsov J, Neuvonen L-K, Korpio T, Uldum SA, Mentula S, Putus T, Tran Minh NN, Martimo KP. Two Legionnaires' disease cases associated with industrial waste water treatment plants: a case report. BMC Infect Dis 2010; 10:343.

4. Miragliotta G, Del Prete R, Sabato R, Cassano A, Carnimeo N. Legionellosis associated with artesian well excavation. Eur J Epidemiol 1992; 8(5):748-749.

5. Fry AM, Rutman M, Allan T, Scaife H, Salehi E, Benson R, Fields B, Nowicki S, Parrish MK, Carpenter J, Brown E, Lucas C, Horgan T, Koch E, Besser RE. Legionnaires' disease outbreak in an automobile engine manufacturing plant. J Infect Dis 2003; 187(6):10151018.

6. Caylà JA, Maldonado R, González J, Pellicer T, Ferrer D, Pelaz C, Gracia J, Baladrón B, Plaséncia A; Legionellosis study group. A small outbreak of Legionnaires' disease in a cargo ship under repair. Eur Respir J 2001; 17(6):1322-1327.

7. Bellido-Blasco JB, Pelaz-Antolín C, Delas-González MA, Sarrión-Martínez J, Moreno-Muñoz MR, Herrero-Carot C. Agregación de casos de neumonía por legionella en trabajadores relacionados con la industria de la cerámica en Castellón en 2006. Rev Esp Salud Pública 2008; 82(1):111-116.

8. Pastoris MC, Greco D, Cacciottolo JM, Vassallo A, Grech A, Bartlett CL. Legionnaires' disease on an oil drilling platform in the Mediterranean: a case report. Br J Ind Med 1987; 44(9):645-646.

9. Gregersen P, Grunnet K, Uldum SA, Andersen BH, Madsen $H$. Pontiac fever at a sewage treatment plant in the food industry. Scand J Work Environ Health 1999; 25(3):291-295.

10. Appleby PH. ABC of work related disorders. Building related illnesses. BMJ 1996; 313(7058):674-647.

11. Isozumi R, Ito Y, Ito I, Osawa M, Hirai T, Takakura S, Iinuma Y, Ichiyama S, Tateda K, Yamaguchi K, Mishima M. An outbreak of Legionella pneumonia originating from a cooling tower. Scand J Infect Dis 2005; 37(10):709-711.

12. Morton S, Bartlett CL, Bibby LF, Hutchinson DN, Dyer JV, Dennis PJ. Outbreak of legionnaires' disease from a cooling water system in a power station. $\mathrm{Br} \mathrm{J}$ Ind Med 1986; 43(9):630-635.

13. Wanden-Berghe C, Sanz-Valero J. Systematic reviews in nutrition: standardized methodology. Br J Nutr 2012; 107(Supl. 2):S3-7.

14. Vandenbroucke JP, Von Elm E, Altman DG, Gøtzsche PC, Mulrow CD, Pocock SJ, Poole C, Schlesselman JJ, Egger M; STROBE Initiative. Strengthening the reporting of observational studies in epidemiology (STROBE): explanation and elaboration. Epidemiology 2007; 18(6):805-835.
15. Girod JC, Reichman RC, Winn WC, Klaucke DN, Vogt RL, Dolin R. Pneumonic and nonpneumonic forms of legionellosis. The result of a common-source exposure to Legionella pneumophila. Arch Intern Med 1982; 142(3):545-547.

16. Castor ML, Wagstrom EA, Danila RN, Smith KE, Naimi TS, Besser JM, Peacock KA, Juni BA, Hunt JM, Bartkus JM, Kirkhorn SR, Lynfield R. An outbreak of Pontiac fever with respiratory distress among workers performing high-pressure cleaning at a sugar-beet processing plant. J Infect Dis 2005; 191(9):1530-1537.

17. Centro Nacional de Epidemiología. Legionelosis: situación general y evolución de la enfermedad en España: datos de la Red Nacional de Vigilancia Epidemiológica (actualización noviembre 2010). Madrid: Instituto de Salud Carlos III; 2010.

18. Centro Nacional de Epidemiología. Brotes de legionelosis notificados a la Red Nacional de Vigilancia Epidemiológica: años 1999-2011. Madrid: Instituto de Salud Carlos III; 2012.

19. Centro Nacional de Epidemiología. Brotes de legionelosis notificados a la Red Nacional de Vigilancia Epidemiológica: años 1999-2009. Madrid: Instituto de Salud Carlos III; 2010.

20. Rowland M. Basic data on hearing levels of adults 2574 years. United States, 1971-75. Vital Health Stat 11 1980; (215):i-vi, 1-49.

21. Armstrong CW, Miller GB. A 1949 outbreak of Pontiac fever-like illness in steam condenser cleaners. Arch Environ Health 1985; 40(1):26-29.

22. Domingo-Pueyo A, Sanz-Valero J, Wanden-Berghe C. Effects of occupational exposure to chromium and its compounds: a systematic review. Arch Prev Riesgos Laborales 2014; 17(3):142-153.

23. O'Keefe NS, Heinrich-Morrison KA, McLaren B. Two linked cases of legionellosis with an unusual industrial source. Med J Aust 2005; 183(9):491-492.

24. Williams P, Liu W-K, Coetzee N, Boulton L. Legionella colonization of powder paint pretreatment tunnels. Ann Occup Hyg 2010; 54(4):475-476.

25. Esteve Pardo M, Casas García I, Pecero Cacho S, Sabrià Leal M. Legionellosis como enfermedad profesional. Med Clínica (Barc) 2008; 131(11):439.
Artigo apresentado em 06/03/2016

Aprovado em 05/06/2017

Versão final apresentada em 07/06/2017 